\title{
THE OPTICAL COUNTERPART OF SAX J1808.4-3658 IN QUIESCENCE: EVIDENCE OF AN ACTIVE RADIO PULSAR?
}

\author{
L. Burderi, ${ }^{1}$ T. Di Salvo, ${ }^{2}$ F. D'Antona, ${ }^{1}$ N. R. Robba, ${ }^{2}$ and V. Testa ${ }^{1}$
}

\section{RESUMEN}

La contraparte óptica del pulsar binario SAX J1808.4-3658 que emite en rayos X con un período de milisegundos. ha sido detectada durante su estapa de quietud a V = 21.5 mag; lo cual es inconsistente con la débil emisión de la estrella compañera. Proponemos que la emisión en el óptico de este sistema durante su etapa de quietud es debida a la irradiación de la estrella compañera y a un disco de acreción permanente provocado por la energía rotacional producida por el rápido giro de la estrella de neutrones que actúa como un dipolo magnético rotante (radio pulsar). En este escenario, la compañera se comporta como un bolómołro, reprocesando en el óptico parte de la potencia emitida por el pulsar. La fracción reprocesada depende sólo de parámetros conocidos del sistema binario; la temperatura de cuerpo negro asociada a la estrella compañera puede predecirse y ratificarse con observaciones. Nuestros cálculos indican que las magnitudes ópticas observadas son totalmente compatibles con esta hipótesis. En el caso de que esto sea así, la luminosidad observada èn el óptico puede ser la primera evidencia de que hay un radio pulsar activo en este sistema en quictud.

\section{ABSTRACT}

The optical counterpart of the binary millisecond X-ray pulsar SAX J1808.4-3658 during quiescence was detected at $\mathrm{V}=21.5 \mathrm{mag}$, inconsistent with intrinsic enission from the fain companion star. We propose that the optical emission from this system during quiescence is due to the irradiation of the companion star and a remnant accretion disk by the rotational energy released by the fast spinning neutron star, switched on, as magneto-dipole rotator (radio pulsar). In this scenario the companion behaves as a bolometer, reprocessing in optical part of the power emitted by the pulsar. The reprocessed fraction depends only on known binary parameters. Thus the blackbody temperature of the companion can be predicted and compared with the observations. Our computations indicate that the observed optical magnitudes are fully consistent with this hypothesis. In this case the observed optical luminosity may be the first evidence that a radio pulsar is active in this system in quiescence.

\section{Key Words: ACCRETION, ACCRETION DISCS — STARS: INDIVIDUAL (SAX J1808.4-3658) - STARS: NEUTRON - X-RAYS: STARS}

\section{INTRODUCTION}

In 1998, the idea that neutron stars (NSs) in low mass X-ray binaries (LMXBs) were spinning at millisecond periods was spectacularly demonstrated by the discovery of coherent X-ray pulsations at $P_{\text {spin }} \sim 2.5 \mathrm{~ms}$ in SAX J1808.4-3658, a transient source with an orbital period $P_{\text {orb }}=2 \mathrm{~h}(\mathrm{Wij}-$ nands \& van der Klis 1998). In the last few months four other X-ray sources have been discovered in which coherent $\mathrm{X}$-ray pulsations in the millisecond range have been found (see Wijnands 2003 for a review). We are, therefore, facing a new class of astronomical objects, the Millisecond X-ray Pulsars (MSXPs) that could constitute the longed for bridge between the accretion-powered (X-ray pul-

\footnotetext{
${ }^{1}$ Osservatorio Astronomico di Roma, Italy.

${ }^{2}$ Dipartimento di Scienze Fisiche ed Astronomiche, Università di Palermo, Italy.
}

sators) and the rotation-powered (Millisecond Radio Pulsar, hereafter MSP) NS sources. as foreseen in the so-called recycling scenario (see e.g. Bhattacharya \& van den Heuvel 1991, for a review), according to which the former are the progenitors of the latter.

The MSXPs are all X-ray transients, which are usually found in a quiescent state, with X-ray luminosities in the range $10^{32}-10^{33} \mathrm{ergs} / \mathrm{s}$. On occasions they exhibit outbursts, during which the luminosity increases to $\sim 10^{36}-10^{38} \mathrm{ergs} / \mathrm{s}$ and their behavior closely resembles that of persistent LMXBs. In these sources from outburst to quiescence the mass transfer rate decreases up to five orders of magnitude and. correspondingly, the magnetospheric radius, $R_{\mathrm{AI}}$. expands by a factor $\sim 30$ during quiescence. Accretion onto a spinning magnetized $\mathrm{NS}$ is centrifugally inhibited once $R_{\mathrm{M}}$ expands beyond the corotation radius, $R_{\mathrm{CO}}=1.5 \times 10^{6} \mathrm{~m}^{1 / 3} \mathrm{P}_{-3}^{2 / 3} \mathrm{~cm}$ ( $m$ is the $\mathrm{NS}$ 
mass in solar masses, $P_{-3}$ is the NS spin period in milliseconds), the radius at which the Keplerian angular frequency of the orbiting matter equals to the NS spin. If $R_{\mathrm{M}}$ further expands beyond the lightcrlinder radius, $R_{\mathrm{LC}}=4.8 \times 10^{6} P_{-3} \quad \mathrm{~cm}$, where an object corotating with the NS attains the speed of light, the NS becomes generator of magnetodipole radiation and relativistic particles. For spin periods up to few milliseconds, $R_{\mathrm{M}} \geq R_{\mathrm{LC}}>R_{\mathrm{CO}}$ in quiescence. Therefore, in this phase accretion is certainly inhibited and is likely that a magneto dipole emitter switches on (as predicted by some authors, see e.g. Stella et al. 1994; Burderi et al. 2001), causing the NS to turn-on as a MSP until a new outburst episode pushes $R_{\mathrm{M}}$ back, close to the NS surface, quenching radio emission and initiating a new accretion phase.

In this paper we show that the hypothesis that a magneto-dipole rotator is active in these systems during quiessence can explain the puzzling optical comnterpart of SAX J1808.4 3658 during quicscence. The optical cmission and morulation during quiescence is therefore the first (indirect) evidence that a magneto-dipole rotator (i.e. a radio pulsar) is active in these systems during quiescence, a key phase for understanding the evolution of this class of objects.

\section{THE OPTICAL COUNTERPART TO SAX J1808.4. 3658}

The optical counterpart of SAX J1808.4-3658 was idcntified by Roche et al. (1998) with the variable star V4584 Sagittarii, a very low mass star (probably a low-mass, irradiation-bloated, brown dwarf; Bildsten (t Chakrabarty 2001). Homer et al. (2001) reported on high time resolution CCD photometry of this optical counterpart observed when the X-ray source was in quiesserucr. The optical component was detected at $V^{\circ} \sim 21.5$ mag. In these data $\mathrm{a} \sim 6 \%$ semi-amplitude modulation at the 2 -hr orbital period of the system is significantly detected. The photometric minimum is found when the companion star lies between the pulsar and the observer and the shape of the modulation is approximately sinusoidal, similar to what is observed during outbursts. The measured optical luminosity in quiescence is $\sim 10^{31} \mathrm{erg} \mathrm{s}^{-1}$ (Homer et al. 2001), inconsistent with the faint intrinsic emission expected from a $\leq 0.14 M_{\odot}$ companion (Chakrabarty \& Morgan 1998). Also the lack of double-humped morphology, due to an ellipsoidal modulation, again excludes the direct optical emission from the companion as the origin of the observed optical flux and modulation. This lead Homer et al. (2001) to interpret the observed optical flux as emission from an accretion disk with an accretion rate of $10^{-11} M_{\odot} /$ yr. However the rxpected X-ray luminosity in this case $\left(\sim 10^{34}-10^{35}\right.$ $\mathrm{erg} / \mathrm{s})$ is incompatible with the measured $X$-ray quiescent luminosity of this source $\left(5 \times 10^{31} \mathrm{erg} \mathrm{s}^{-1}\right.$, Campana et al. 2002). We show that these difficulties are solved if we make the case that actually the irradiation of the companion star and /or the remnant disk is due to illumination from the radio pulsar, which may switch on during quiescence, once the magnetospheric radius is pushed beyond the light cylinder radius.

\section{IRRADIATION OF THE DISK AND COMPANION STAR BY ROTATING MAGNETO-DIPOLE EMISSION}

It has been demonstrated that a rotating magnetic dipole in vacuum emits electromagnetic dipole radiation. Also, a wind of relativistic particles associated with magnetospheric currents along the field lines is expected to arise in a rotating NS (e.g. Goldreich \& Julian 1969). In the hypothesis that the magneto-dipole rotator is active in SAX J1808.43658 during quiescence, we can evaluate the power of the pulsar beam and, consequently the irradiation luminosity. The magnetic field of SAX J1808.4-3658 is constrained in the quite narrow range $(1-5) \times 10^{8}$ Gauss (see Di Salvo \& Burderi 2003). Adopting this magnetic field we can calculate the spin-down energy loss of the pulsar: $L_{P S R}=\left(2 / 3 c^{3}\right) \mu^{2} \omega^{4}=$ $3.85 \times 10^{35} P_{-3}^{-4} \mu_{26}^{2} \mathrm{ergs} / \mathrm{s} \sim(1-25) \times 10^{34} \mathrm{ergs} / \mathrm{s}$, where $\omega$ is the rotational frequency of the NS, $\mu$ is the NS magnetic moment, and $\mu_{26}$ is the NS magnetic moment in units of $10^{26}$ Gauss $\mathrm{cm}^{3}$.

In the hypothesis that the pulsar beam heats a remnant accretion disk and/or a side of the companion star and assuming isotropic emission, we can evaluate the fraction of the irradiation luminosity that will be intercepted and reprocessed by the disk and the companion star, respectively. For the accretion disk this fraction, $f_{D}$, is given by the projected area of the disk as seen by the central source, $2 \pi R \times 2 H(R)$ (where $R$ is the disk outer radius and $H(R)$ is the disk semi-thickness at $R$ ) divided by the total area, $4 \pi R^{2}$. Adopting a standard ShakuraSunyaev disk model (Shakura \& Sunyaev 1973), we find: $f_{D} \sim(1.5-2.1) \times 10^{-2}$, using a reasonable value of the viscosity parameter, $\alpha=0.1$, and adopting $\dot{M}_{-10}=0.1-1$ (note that $f_{D}$ depends weakly on the mass accretion rate). The fraction $f_{C}$ of the pulsar spir-down luminosity reprocessed by the companion star and emitted in the optical band can be written as: $f_{C}=2 \pi a^{2}(1-\cos \theta) /\left(4 \pi a^{2}\right)$, where $a$ is the the orbital separation and $\theta$ is the angle subtended by 
the companion star as seen from the central source: we obtain: $f_{C} \sim 1.1 \times 10^{-2}$.

If both the outer accretion disk and the companion star are reprocessing the pulsar spin-down luminosity, the total fraction of this luminosity that will be intercepted and reprocessed is: $f=f_{D}+f_{C} \sim$ $3.1 \times 10^{-2}$, giving an optical reprocessed luminosity of $\sim 3 \times 10^{32} \mu_{26}^{2} \mathrm{ergs} / \mathrm{s}$. We have calculated the predicted apparent magnitudes in three optical bands, which are perfectly in agreement with the measures reported by Homer et al. (2001) in the same optical bands. The results are shown in Figure 1.

In our model, the optical inodulation should again be caused by the heated side of the companion star, in agreement with the lack of cllipsoidal variations that should be expected in the intrinsic optica! emission if the companion star fills its Roche lobe, as required in this system.

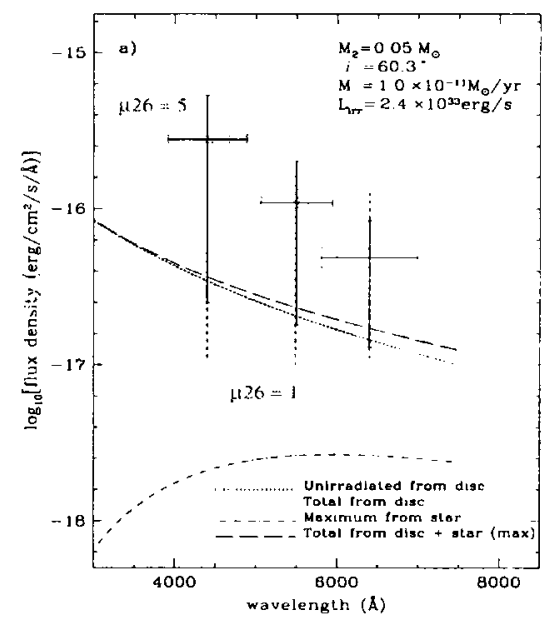

Fig. 1. Optical (BVR) photometry of V4580 Sgr (SAX J1808.4-3658 optical counterpart, points with error bars), with model spectra from various (irradiated) disc/star emission components (as labelled). The dashed error bars indicate the maximum and minimum fluxes exhibited during the $2-\mathrm{h}$ modulation cycle, to be compared with the variation between total from irradiated disc (solid line) and total from irradiated disc plus star (long dashed line). From Homer et al. (2001). The (red) vertical dashed lines indicate the range of magnitudes predicted on the basis of the magneto-dipole rotator model described in the text assuming a NS magnetic moment in the range $(1-5) \times 10^{26}$ Gauss $\mathrm{cm}^{3}$.

\section{DISCUSSION AND CONCLUSIONS}

We have proposed here that the optical counterpart of SAX J1808.4-3658 during quiescence can be due to irradiation by the release of rotational energy by the fast spinning NS, switched on as a magneto-dipole rotator (radio pulsar) once the magnetospheric radius is pushed beyond the light cylinder radius during quiescence. Our computations in- dicate that the optical magnitudes are fully consistent with this hypothesis. The scenario we propose does not require a high mass accretion rate through the disk nor a quiescent X-ray luminosity of $10^{33}$ $\mathrm{ergs} / \mathrm{s}$, because the luminosity for the reprocessing is supplied by the rotational energy of the pulsar. $L_{\mathrm{PSR}} \sim(1-25) \times 10^{34} \mathrm{ergs} / \mathrm{s}$. Also, we can explain why a similar semi-amplitude of $\sim 6 \%$ is observed in the optical modulation both in outburst and in quiescence, since the reprocessor geometry in our scenario does not substantially change between outburst and quiescence.

The presence of an artive magneto-dipole emitter during quiescence in SAX J1808.4-3658 as required in this scenario, suggest that SAX J1808.43658 should show radio pulsations in quiescence. Indeed, despite thoroughly searcherl in radio cluring its $X$-ray quiescent phàse, no pulsed radio emission has been detected from this source up to now (Gaensler et al. 1999; Burgay et al. 2003). The lack of radio detection can be caused by the presence of a strong wind of matter emanating from the system. i.e. the mass released by the companion star swept away by the radiation pressure of the pulsar, as predicted in the so-called radio-ejection model (Burderi et al. 2001). This means that SAX J1808.4-3658 may show radio pulsations in quiescence when observed at frequencies higher than the standard 1.4 $\mathrm{GHz}$ (the frequency at which radio pulsars are normally searched), where the free-free absorption is less severe.

\section{REFERENCES}

Bhattacharya, D., \& van den Heuvel, E.P.J. 1991. Physics Reports, 203, 1

Bildsten, L., \& Chakrabarty, D. 2001, ApJ, 557, 292

Burderi, L., et al. 2001, ApJ, 560, L71

Burgay, M., et al. 2003, ApJ, 589, 902

Campana, S., et al. 2002, ApJ, 575, L15

Chakrabarty, D., \& Morgan, E.H. 1998, Nature, 394. 346

Di Salvo, T., \& Burderi, L. 2003, A\&A, 397, 723

Gaensler, B.M., Stappers, B.W., Getts. T.J. 1999, ApJ. $522, \mathrm{~L} 117$

Goldreich P., \& Julian, W.H. 1969, ApJ, 157, 869

Homer, L., Charles, P.A., Chakrabarty, D., van Zyl, L. 2001, MNRAS, 325, 1471

Roche, P., et al. 1998, IAUC N.6885, 1

Shakura, N.I., \& Sunyaev, R.A. 1973, A\&A. 24. 337

Stella, L., Campana, S., Colpi, M., Mereghetti, S., Tavani, M. 1994, ApJ, 423, L47

Wijnands, R., \& van der Klis, M. 1998, Nature, 394. 344

Wijnands, R., Proc. of the Symposium "The Restless High-Energy Universe", E.P.J. van den Heuvel, J.J.M. in "t Zand, and R.A.M.J. Wijers Eds. (astroph/0309347) 